\title{
Merging $\boldsymbol{W} \boldsymbol{W}$ and $\boldsymbol{W} \boldsymbol{W}+$ jet with MINLO
}

\author{
Keith Hamilton, ${ }^{a}$ Tom Melia, ${ }^{b, c}$ Pier Francesco Monni, ${ }^{d}$ Emanuele $\mathbf{R e}^{e}$ \\ and Giulia Zanderighi ${ }^{d, f}$ \\ ${ }^{a}$ Department of Physics and Astronomy, University College London, \\ London, WC1E 6BT, U.K. \\ ${ }^{b}$ Berkeley Center for Theoretical Physics, University of California, \\ Berkeley, CA 94720, U.S.A. \\ ${ }^{c}$ Theoretical Physics Group, Lawrence Berkeley National Laboratory, \\ Berkeley, CA 94720, U.S.A. \\ ${ }^{d}$ Rudolf Peierls Centre for Theoretical Physics, University of Oxford, \\ Oxford OX1 3NP, U.K. \\ ${ }^{e}$ LAPTh, Université Savoie Mont Blanc, CNRS, \\ B.P.110, Annecy-le-Vieux F-74941, France \\ ${ }^{f}$ Theoretical Physics Department, CERN, \\ Geneva, Switzerland \\ E-mail: keith.hamilton@ucl.ac.uk, tmelia@lbl.gov, \\ pier.monni@physics.ox.ac.uk, emanuele.re@lapth.cnrs.fr, \\ giulia.zanderighi@cern.ch
}

ABSTRACT: We present a simulation program for the production of a pair of $W$ bosons in association with a jet, that can be used in conjunction with general-purpose shower Monte Carlo generators, according to the POWHEG method. We have further adapted and implemented the MINLO' method on top of the NLO calculation underlying our $W^{+} W^{-}+$jet generator. Thus, the resulting simulation achieves NLO accuracy not only for inclusive distributions in $W^{+} W^{-}+$jet production but also $W^{+} W^{-}$production, i.e. when the associated jet is not resolved, without the introduction of any unphysical merging scale. This work represents the first extension of the MINLO' method, in its original form, to the case of a genuine underlying $2 \rightarrow 2$ process, with non-trivial virtual corrections.

KeYwORDS: NLO Computations, Jets

ARXiv EPRINT: 1606.07062 


\section{Contents}

1 Introduction 1

2 Method and technical details $\quad 4$

2.1 NLOPS construction 4

2.2 NLO calculation validation 5

$2.3 \mathrm{MINLO}^{\prime}$ for general jet-associated colourless particle production processes $\quad 6$

$\begin{array}{llr}3 & \text { Phenomenological results } & 8\end{array}$

3.1 Comparison of WW and WWJ-MinLO' generators 8

$\begin{array}{lll}3.1 .1 & \text { Total cross sections } & 10\end{array}$

$\begin{array}{lll}3.1 .2 & \text { Inclusive observables } & 11\end{array}$

$\begin{array}{lll}3.1 .3 & \text { Jet associated production } & 14\end{array}$

4 Conclusion and outlook $\quad 17$

\section{Introduction}

Run II at the LHC will further explore physics at the scale of electroweak symmetry breaking and continue the search for new phenomena in the TeV energy range. Within this programme, major attention will be paid to sharpening measurements of Higgs boson properties, searching for direct and indirect signals of new particles, and excluding and curtailing the form of proposed physics beyond the standard model. For all of these endeavours, the QCD production of pairs of electroweak (EW) gauge bosons is an important process over which to exert theoretical and experimental control. Weak boson pair production constitutes a major background in Higgs boson analyses, as well as in direct searches for new particles decaying into jets, leptons and/or missing energy. Furthermore, precision measurements of these processes translate to indirect bounds on new physics at higher energies than are directly accessible, e.g. through setting constraints on the allowed size of anomalous trilinear gauge interactions.

Studies of $W^{+} W^{-}$hadroproduction have been carried out both at the Tevatron ${ }^{1}$ and the $\mathrm{LHC},{ }^{2}$ in which limits on anomalous triple gauge couplings were derived. For LHC Run I measurements, experimental uncertainties are at the level of $7-8 \%$, and are dominated by systematics. One important source of uncertainty occurs when both $W$ bosons decay leptonically: the final-state contains two neutrinos, whose missing momenta prevent a full reconstruction of the event kinematics - in particular the momenta of the $W$ bosons. The lack of any resonant $W$ mass peaks leads to a greater sensitivity of experimental analyses to

\footnotetext{
${ }^{1}$ See e.g. [1-3] and references therein.

${ }^{2}$ See e.g. $[4,5]$ and references therein.
} 
the theoretical modelling of this process than would otherwise be the case, be it as a signal, or a background. The kinematic distributions which are used as experimental handles have a greater susceptibility to QCD radiative corrections; the uncertainty connected to this modelling is a contributing factor in the experimental systematic error estimate. Separately, in order to isolate $W^{+} W^{-}$final-states from backgrounds, in particular those due to $t \bar{t}$ and $t W$ production, experimental analyses categorise events according to their jet multiplicity, in so-called jet-binned/jet-vetoed analyses. Such event selections are employed in the study of both QCD continuum $W^{+} W^{-}$pair production, as well as Higgs boson production in the $H \rightarrow W^{+} W^{-}$channel (in which context the former signal process plays the rôle of an irreducible background). In both analyses, the fact that the signal definition includes cuts on the presence of associated jets also implies a potentially marked sensitivity to higher order QCD effects: in the study of continuum QCD $W^{+} W^{-}$production the ensuing uncertainty significantly contributes to the extrapolation to the total cross-section. All considered, the above experimental issues point to the importance of flexible, high-accuracy, and fully realistic, theoretical predictions for $W^{+} W^{-}$and jet-associated $W^{+} W^{-}$production processes.

The level of theoretical precision with which hadronic $W$-pair production is known has seen truly remarkable progress in recent years. Partonic QCD calculations for $p p \rightarrow W^{+} W^{-}$ have evolved from stable- $W$ approximations at LO [6] and NLO $[7,8]$, to much more sophisticated treatments, incorporating spin correlation and off-shell effects in $W$ decays, all at NLO [9-11]. The latter are available as flexible public computer codes, such as MCFM [12]. The NLO calculation of $W$-pair production in association with a jet, including $W$ decays, was first carried out almost ten years ago [13-15], with the dijet case following in 2011 [16]. Gluon initiated contributions to $W$-pair hadroproduction were calculated in refs. [17-20], the contribution due to interference with Higgs boson production being later taken into account in ref. [21]. The leading order gluon fusion contribution to jet-associated $W$-pair production was first computed in ref. [22]. More recently, in the last couple of years, NNLO predictions for $W$-pair production have become available, for the case of stable bosons [23], using the two loop helicity amplitudes of ref. [24]. Off-shell two-loop amplitudes have also been computed lately, both for quark-antiquark collisions [25, 26], and gluon fusion $[27,28]$. The latter results have been used in determining the NLO corrections to $g g \rightarrow W^{+} W^{-}$[29]. The quark-antiquark two-loop amplitudes have recently been used to present fully differential NNLO predictions [30]. Finally, at fixed order, we note that NLO electroweak corrections to $W$-pair production are also known [31-33], even including a full off-shell treatment of the $W$ decays [34].

In the context of resummed QCD calculations, transverse momentum and threshold resummations for this process have been studied in refs. [35-38] and ref. [39] respectively. The effects of a jet veto resummation were considered by several groups [40-43], in part triggered by a discrepancy between the measured and predicted cross sections at the LHC.

Monte Carlo event generators matching NLO $W$-pair production calculations to parton showers (NLOPS) have been publicly available for around a decade. Indeed this process was the subject of the pioneering proof-of-concept work demonstrating the MC@NLO NLOPS matching formalism [44]. Alternative NLOPS implementations of this process, using different methods and approximations, were subsequently implemented in the HeRwiG ++ [45], 
Sherpa [46] and Powheg-Box [47, 48] packages. The original Herwig ++ implementation has recently been superseded by a version including single-resonant and gluon induced contributions, in the new HERWIG7 framework [49, 50]. NLOPS simulations of $W$-pair production and $W$-pair production in association with a jet have been merged by the OpENLoops+SHERPA group [51] according to the MePs@NLO [52, 53] merging scheme. The AMC@NLO team have also presented state-of-the-art simulations of NLO weak boson pair production in ref. [54], which can be automatically merged with higher order jet multiplicities according to the FXFx multi-jet merging method [55, 56]. All of the above simulations include full leptonic decay kinematics, with the latter two simulations (employing NLO merging) also incorporating $g g$ initiated contributions.

In this paper we extend and apply the MINLO' technique of ref. [57], to deliver a NLO calculation of jet-associated $W$-pair production, which is simultaneously NLO accurate in the description of 0-jet quantities. In our underlying NLO calculation the $W$ bosons are allowed to decay either hadronically or leptonically. In the hadronic case, we incorporate the NLO QCD correction to the decay only inclusively. We employ fully off-shell matrix elements, including singly-resonant contributions, but we omit the loop-mediated $g g$ channel in our simulation. The latter is separately finite and can be accounted for straightforwardly via, e.g., the GG2Ww event generator $[20,58]$ (as employed by ATLAS and CMS). The enhanced Minlo' computation is implemented within the PowHEG-Box Nlops framework [59-61], such that approximate higher order, higher twist, and non-perturbative QCD effects can be accounted for (parton shower, hadronization and underlying event), rendering a realistic description of the final-state. The latter class of corrections can have a non-negligible impact on many observables; in particular, for jet-binned cross sections, where they contribute to migrations between bins, their effects can be sizable, as noted in, e.g., ref. [62].

We reiterate that not only is our single MINLO' calculation of jet-associated $W$-pair production capable of populating the phase space of the 0 -jet region, it can be formally proved that the predictions which it yields for 0-jet quantities are also NLO accurate. This is in difference to the earlier-mentioned multi-jet merged NLOPS simulations, which essentially partition phase space into jet bins, whose 'size' is set by a new merging scale parameter, with each bin being populated by events from an NLOPS simulation with the corresponding jet multiplicity.

The current work is novel theoretically, in so far as it represents the first application of the original MinLO' method to a genuine $2 \rightarrow 2$ colour singlet process at the lowest order, ${ }^{3}$ wherein the virtual $(V)$ and Born $(B)$ contributions are not proportional to each other. The ratio $V / B$ enters the process-dependent part of the NNLL resummation coefficient $B_{2}$, whose inclusion in the Minlo Sudakov form factor is mandatory for yielding NLO accuracy also in the description of 0-jet quantities. In the original MinLO' works on Higgs and Drell-Yan production the ratio $V / B$ is just a constant (since these are effectively $2 \rightarrow 1$ processes) while the extension to the present case requires a procedure to compute the process-dependent $B_{2}$ term for the production of a generic colour-singlet system.

\footnotetext{
${ }^{3}$ An alternative extension of MiNLO' was given in ref. [63].
} 
The MinLO' implementation presented here can be readily promoted to a NNLOPS simulation of $W^{+} W^{-}$production following the same procedure employed to build NNLOPS $H$ [64, 65], $Z$ [66], and $H W$ [62] generators. The present work can be regarded as a main theoretical step towards such a NNLOPS simulation of $W^{+} W^{-}$production.

An NNLOPs generator could also be achieved through matching an NNLO + NNLL' resummed calculation of this process to a parton shower using the GENEvA matching formalism [67-70]. In addition, it would appear to be a straightforward matter to merge the same NNLO calculation with a parton shower according to the UN ${ }^{2}$ LOPS prescription [71, 72].

The paper is structured as follows. In section 2 we give details on the construction of our underlying NLO calculation for $W$-pair production in association with a jet, as well as details on the validation of our implementation. We then proceed to exemplify the extension of the MINLO' method to a generic colour-singlet process. Many aspects of the MiNLO' approach follow unchanged from refs. [57, 73], and so we focus on presenting the key differences and their practical implementation. Section 3 presents a phenomenological study of kinematic distributions for the decay mode of the $W$-bosons to $e^{+} \nu_{e} \mu^{-} \bar{\nu}_{\mu}$. We summarize our findings and conclude in section 4 . We have made our simulation publicly available within the POWHEG-Box code. ${ }^{4}$

\section{$2 \quad$ Method and technical details}

In this section we first give all details concerning the construction of the pure NLOPS simulation of jet-associated $W$-pair production (henceforth WWJ), including the treatment of heavy fermions and the CKM matrix. We subsequently detail the validation of this construction. Following this, we go on to describe how we have modified and extended the original MINLO' method, such that our WWJ-MinLO simulation also recovers NLO accurate results for 0 -jet and inclusive $W$-pair production observables (henceforth WW).

\subsection{NLOPS construction}

We have generated Born and real matrix elements using the PowHEG-Box interface to MADGRAPH 4 [74] developed in ref. [75]. The virtual matrix elements have been obtained using GoSAm 2.0 [76]. Our code is based on matrix elements for the following Born subprocesses and all of their associated NLO counterparts: ${ }^{5}$

$$
q \bar{q} \rightarrow e^{+} \nu_{e} \mu^{-} \bar{\nu}_{\mu} g, \quad q g \rightarrow e^{+} \nu_{e} \mu^{-} \bar{\nu}_{\mu} q, \quad \bar{q} g \rightarrow e^{+} \nu_{e} \mu^{-} \bar{\nu}_{\mu} \bar{q} .
$$

Hence, while we refer to our simulation as being one of WWJ production, we do in fact include full spin correlations and all related off-shell and single-resonant contributions. That is, while no $Z Z$ or $Z \gamma$ contributions exist for the unequal flavour choice, we include all other $Z, W$ or $\gamma$ intermediate exchanges. We have not included the Higgs contribution.

We have chosen to work throughout in the four-flavour scheme (4FNS), as employed, for instance, in the NNLO calculations of $W^{+} W^{-}$production in refs. [23, 30]. Thus, we do

\footnotetext{
${ }^{4}$ Instructions to download the code can be obtained at http://powhegbox.mib.infn.it.

${ }^{5}$ In section 2.2 we will also discuss the impact of removing the gauge-invariant set of fermionic loop corrections.
} 
not include effects from third generation quarks. In doing so we most easily avoid significant complications that affect 5FNS calculations, in particular those due to the opening of resonant $t W$ and $t \bar{t}$ channels at $\mathcal{O}\left(\alpha_{\mathrm{S}}\right)$ and $\mathcal{O}\left(\alpha_{\mathrm{S}}^{2}\right)$. The latter resonant top-pair contributions enhance the inclusive $p p \rightarrow W^{+} W^{-}+X$ cross section by a factor of 4 (8) at the 7 (14) $\mathrm{TeV}$ LHC [23], but they give rise to experimentally separable signatures. This necessitates a theoretical definition of $W^{+} W^{-}$hadroproduction wherein the top contributions are subtracted, analogous to that employed for experimental measurements of this process. This issue has been studied in ref. [23], where it was shown that by an appropriate removal of the resonant top contributions in the 5FNS case, the 4FNS (with third generation quarks omitted) and the 5FNS NNLO predictions agree at the level of $1-2 \%$.

For the $W$ boson decays in our program, the user can select leptonic decay modes (summed over generations, or for just a single generation), and/or hadronic decay modes (summed over all kinematically allowed flavours). The chosen decay channels are then taken into account when generating Les Houches events. When a leptonic decay into more than one generation is selected, we randomly generate the lepton flavour, accounting for the relevant combinatorial factors. For a hadronic decay, an up or charm quark is chosen at random and the related down-type quark is selected with a probability given by the associated Cabibbo matrix element squared. When writing out the corresponding part of the Les Houches event record, we assign the quark and anti-quark originating from the decay of the $W$ to the same colour line. Furthermore, for hadronic decays we include the NLO correction to the inclusive $W$-hadronic branching ratio. ${ }^{6}$ In the case of decays to leptons of the same family, we do not include double resonant $Z Z$ production with one boson decaying to leptons and one invisibly. In fact, we consider the latter as being part of the $Z Z$ production process, and, as shown in ref. [47], the interference between the $W W$ and $Z Z$ mediated processes is completely negligible.

We defer details of the technical checks performed in the course of assembling this NLO calculation, within the PowHEG-Box framework, to section 2.2, where a full validation of our final WWJ-MinLo generator is given.

\subsection{NLO calculation validation}

The fixed order calculation described in section 2.1 underlying our WWJ-MINLO generator and implemented in the PowHEG-Box framework has been cross-checked at leading order against MADGRAPH5_AMC@NLO [56], and at the NLO level against the independent WWJ code of ref. [77]. Beside point-by-point checks of the matrix elements, total cross sections and differential distributions were compared and found to agree very satisfactorily in all cases.

In the calculation of one-loop matrix elements we include fermion loops. However, adding the latter slows down the event generation significantly. In our main validation work of the full WWJ-MinLO generator, described in section 3, we examined numerous distributions, probing a wide range of kinematic configurations, and did not find any distribution in which there was a statistically significant difference exceeding 1-2\% between

\footnotetext{
${ }^{6}$ In the case of hadronic decays, we neglect t-channel boson exchanges.
} 
results obtained with and without fermionic loop diagrams. We therefore also release a version of the code that omits the gauge-invariant set of fermionic loop corrections.

\subsection{MiNLO' for general jet-associated colourless particle production processes}

Here we describe how to generalize the original MINLO' procedure to deal with general jet-associated colourless particle production, with particular reference given to WWJ. We emphasise similarities and differences relative to the original MinLO' codes [57] addressing jet-associated, single, colourless particle production.

The Minlo' recipe and proof needs as its primary ingredient and starting point an NLO cross section, here the WWJ calculation described above. In general, the latter decomposes into a sum of a part which is finite as the transverse momentum of the colourless system $\left(p_{\mathrm{T}}\right)$ tends to zero, $d \sigma_{\mathcal{F}}$, plus a correspondingly singular part, $d \sigma_{\mathcal{S}}$. The finite piece, $d \sigma_{\mathcal{F}}$, being power suppressed, is essentially a spectator in proofs that MinLO yields NLO accuracy for the inclusive/0-jet process, here $W^{+} W^{-}$production. The singular part of the cross section, differential in the phase-space variables $\Phi$ fully parametrising the underlying $q \bar{q} \rightarrow W^{+} W^{-}$scattering (including $\mathrm{W}$ decays) and the large $\operatorname{logarithm}, L=\ln Q^{2} / p_{\mathrm{T}}^{2}$, here with $Q=m_{W W}$ (the invariant mass of the $W^{+} W^{-}$system), can be obtained by an explicit fixed order calculation, or expanding the NNLL resummed $p_{\mathrm{T}}$ spectrum up to and including $\mathcal{O}\left(\bar{\alpha}_{\mathrm{S}}^{2}\right)$ terms $\left(\bar{\alpha}_{\mathrm{S}}=\alpha_{s} / 2 \pi\right)$. This singular part can be obtained by identifying and replacing all instances of the process-dependent hard function in the Drell-Yan case, with that of $W^{+} W^{-}$production. $^{7}$ The resulting expression for $d \sigma_{\mathcal{S}}$ thus has the form

$$
\frac{d \sigma_{\mathcal{S}}}{d \Phi d L}=\frac{d \sigma_{0}}{d \Phi} \sum_{n=1}^{2} \sum_{m=0}^{2 n-1} H_{n m} \bar{\alpha}_{\mathrm{S}}^{n}\left(\mu_{R}^{2}\right) L^{m}
$$

where the explicit $H_{n m}$ coefficients can be extracted from, for example, the general formulae in appendix A of ref. [63]; they are lengthy and so we do not repeat them here. The processdependent hard function, $H_{1}$, in the $\mathcal{H}_{1}$ terms of [63], is related to the finite part of the renormalized NLO virtual contribution to $W^{+} W^{-}$production, $\mathcal{V}$, as follows:

$$
\mathcal{V}(\Phi)=\frac{1}{\Gamma(1-\epsilon)}\left(\frac{4 \pi \mu^{2}}{Q^{2}}\right)^{\epsilon} \bar{\alpha}_{\mathrm{S}}\left[-\frac{2 C_{F}}{\epsilon^{2}}-\frac{3 C_{F}}{\epsilon}-C_{F} \zeta_{2}+H_{1}(\Phi)\right] \mathcal{B}(\Phi) .
$$

Here, in eq. (2.3), $\mathcal{B}$ is the Born cross section, with the normalization of refs. [60, 61], $\mu$ is the renormalization scale and $\epsilon$ sets the dimensionality, $d$, of spacetime in conventional dimensional regularization $(d=4-2 \epsilon)$. Apart from the one-to-one replacement of these hard function terms, the NLO expression for $d \sigma_{\mathcal{S}}$ is identical to the corresponding formula for the Drell-Yan $p_{\mathrm{T}}$ spectrum. $^{8}$

Having noted the nature of the differences between the singular behaviour of the jetassociated Drell-Yan cross section and that of WWJ, the task of extending the MinLo' method to the latter reduces to that of replacing the $H_{1}(\Phi)$ function everywhere it occurs

\footnotetext{
${ }^{7}$ This statement generalizes trivially to all jet-associated colour singlet production processes.

${ }^{8}$ In a nutshell, physically, this owes to the fact that the underlying primary scattering processes are identical from the point of view of the flow of colour charge, and to the universal character of the infrared QCD corrections which dress them to yield $d \sigma_{\mathcal{S}}$.
} 
in the procedure. Modulo this isolated change in the recipe, the MinLO' implementation and its proofs follow in exactly the same way as before. Indeed, in the procedure itself the $H_{1}(\Phi)$ function only occurs once among the additional components to be layered onto the pure WWJ NLO calculation. Specifically, it only occurs in the process-dependent $B_{2}$ coefficient of the Minlo' Sudakov form factor. Thus, the extension of the Minlo' method to arbitrary colourless particle production processes, which are at lowest order $q \bar{q}$ initiated, consists of generalizing the Sudakov form factor exponent given in the original article [57]:

$$
\log \Delta^{2}\left(Q, p_{\mathrm{T}}\right)=-\sum_{i=1}^{2} \int_{p_{\mathrm{T}}^{2}}^{Q^{2}} \frac{d \mu^{2}}{\mu^{2}} \bar{\alpha}_{\mathrm{S}}^{i}\left(\mu^{2}\right)\left[A_{i} \log \frac{Q^{2}}{\mu^{2}}+B_{i}\right],
$$

with

$$
\begin{aligned}
& A_{1}=2 C_{F}, \quad B_{1}=-3 C_{F}, \quad A_{2}=2 C_{F} K, \\
& B_{2}=-2 \gamma^{(2)}+\bar{\beta}_{0} C_{F} \zeta_{2}+2\left(2 C_{F}\right)^{2} \zeta_{3}+\bar{\beta}_{0} H_{1}(\Phi),
\end{aligned}
$$

and

$$
\begin{aligned}
K & =\left(\frac{67}{18}-\frac{\pi^{2}}{6}\right) C_{A}-\frac{10}{9} n_{f} T_{R}, \quad \bar{\beta}_{0}=\frac{11 C_{A}-4 n_{f} T_{R}}{6} \\
\gamma^{(2)} & =\left(\frac{3}{8}-\frac{\pi^{2}}{2}+6 \zeta_{3}\right) C_{F}^{2}+\left(\frac{17}{24}+\frac{11}{18} \pi^{2}-3 \zeta_{3}\right) C_{F} C_{A}-\left(\frac{1}{12}+\frac{\pi^{2}}{9}\right) C_{F} n_{f} .
\end{aligned}
$$

While the above formulae are specific to the case of processes which are $q \bar{q}$ initiated at the lowest order, the necessary modifications to deal with the $g g$ case are obvious. Hence we see that almost the only change to be implemented in the Drell-Yan MinLO' code components, to enable it for use with WWJ, is the replacement

$$
B_{2} \rightarrow B_{2}-\bar{\beta}_{0} H_{1}^{(\mathrm{DY})}+\bar{\beta}_{0} H_{1}^{(\mathrm{ww})}(\Phi) .
$$

This brings us to the final subtlety. In Drell-Yan processes, which were the subject of the original MINLO' article [57], $H_{1}^{(\mathrm{DY})}$ has no dependence on any kinematics and is just a number:

$$
H_{1}^{(\mathrm{DY})}=C_{F}\left[\pi^{2}-8+\zeta_{2}\right] .
$$

This particularly simple form owes to the fact that Drell-Yan is, from the point of virtual QCD corrections, a single, colourless, particle production process. This is in marked contrast to the general case. Indeed, in $W^{+} W^{-}$production the finite virtual corrections lead to a non-trivial dependence of $H_{1}(\Phi)$ on the kinematics of the underlying hard scattering process which produces the primary $W^{+} W^{-}$system.

To effect the above transformation of $B_{2}$ in eq. (2.9), one first needs a set of WW kinematics, $\Phi$, with which to evaluate the $H_{1}^{(\mathrm{ww})}(\Phi)$ factor. This is not a trivial matter since in MINLO' one has only WWJ Born and virtual configurations, and WWJJ real configurations. For the WWJ Born and virtual contributions we define $\Phi$, event-by-event, by a projection of the WWJ state onto a WW one, using the FKS mapping relevant for 
initial-state radiation in NLO calculations, as described in ref. [60]. ${ }^{9}$ For real emission events, we first apply a projection to the WWJ underlying Born configuration which the POWHEG-Box framework generated the given real configuration from in the first place (also according to an FKS mapping), before projecting a further step back to a WW state in precisely the same way as described for the WWJ Born and virtual configurations. In all cases, in the limit that the total transverse momentum of emitted radiation is small, the effect of the projection on the WW kinematics and its decay products smoothly vanishes. Taking the latter two features as defining criteria for a legitimate projection procedure, any residual ambiguities in their definition will result only in power suppressed corrections, affecting the precise numerical determination of $B_{2}$ safely beyond the level of the $\mathrm{N}^{3} \mathrm{LL}_{\sigma}$ terms which must be controlled ( $B_{2}$ itself already only enters at $\mathrm{N}^{3} \mathrm{LL}_{\sigma}$ order); indeed, for ambiguities in the projection to invalidate the MINLO' simulation they would need to give rise to at least relative $\mathcal{O}(1)$ shifts in the numerical value of $B_{2}$ as $p_{\mathrm{T}} \rightarrow 0$. With these kinematic considerations in hand, we compute the two $H_{1}$ terms in eq. (2.9) by calling their associated PowHEG-Box setvirtual and compborn subroutines [61], whose return values are virtual and born respectively, which, for $q \bar{q}$ initiated colour singlet production processes, we have determined obey the following relation [60, 61]:

$$
\frac{\text { virtual }}{\text { born }}=H_{1}(\Phi)-C_{F} \zeta_{2}-3 C_{F} \log \left(\frac{\mu_{R}^{2}}{\hat{s}}\right)-C_{F} \log ^{2}\left(\frac{\mu_{R}^{2}}{\hat{s}}\right),
$$

$\hat{s}$ being the invariant mass of the final-state particles. For the case of Drell-Yan processes the latter ratio is trivially equal to $C_{F}\left[\pi^{2}-8\right]$ for $\mu_{R}^{2}=\hat{s}$. Finally then in practice we determine $B_{2}^{(\mathrm{ww})}$ event-by-event as

$$
B_{2}^{(\mathrm{WW})}=B_{2}^{(\mathrm{DY})}-\bar{\beta}_{0}\left[C_{F}\left[\pi^{2}-8\right]-\left.\frac{\text { virtual }}{\text { born }}\right|_{\mu_{R}^{2}=\hat{s}} ^{(\mathrm{wW})}\right] .
$$

\section{Phenomenological results}

In this section we undertake a phenomenological study which also serves to exemplify some of the work done to validate our WWJ-MINLO generator, and the improved description that it yields for a variety of important kinematic distributions. To that end, we mainly compare our WWJ-Minlo program to the existing Powheg-Box WW simulation code [47]. The analysis shown in section 3.1 is, however, only a representative summary of a more widereaching comparative study, whose findings are mentioned in the accompanying discussion when relevant.

\subsection{Comparison of $\mathrm{WW}$ and $\mathrm{WWJ}-\mathrm{MINLO}^{\prime}$ generators}

For the purposes of validation and demonstrating the improvements yielded by our WWJMiNLO simulation, we compare and contrast its predictions for a number of kinematic

\footnotetext{
${ }^{9}$ We first boost all momenta to the frame in which the $W^{+} W^{-}$system has zero rapidity. Subsequently we boost all momenta to the frame in which the $W^{+} W^{-}$system has zero transverse momentum, before applying a final boost to the frame where the $W^{+} W^{-}$system has the same rapidity it started with.
} 
quantities of general interest to those of the existing WW POWHEG-Box generator (which we ultimately aim to replace).

In the following we consider only the process $p p \rightarrow e^{+} \nu_{e} \mu^{-} \bar{\nu}_{\mu}+X$ and $13 \mathrm{TeV}$ LHC collisions. We set the $Z$ mass to $91.188 \mathrm{GeV}$ and its width to $2.441 \mathrm{GeV}$. The $W$ mass and width are taken to be $80.149 \mathrm{GeV}$ and $2.0476 \mathrm{GeV}$ respectively. We derive the value of the finestructure constant, according to the so-called $G_{\mu}$-scheme, as being $\alpha_{e m}\left(M_{Z}\right)=1 / 132.507$. The parton distribution functions we have used are the NLO, $n_{f}=4$, NNPDF3.0 [78] set, with the associated running coupling, since we work in the 4FNS. Unless otherwise stated, all predictions shown have been obtained by showering the POwHEG-Box's hardest emission events with Pyтнia 8 [79-81], including hadronization but not multi-parton interaction effects.

Both WW and WWJ-Minlo predictions are obtained with the PowheG-Box bornzerodamp feature activated [61] (see also appendix B of ref. [47]). This flag has the effect of limiting the amount by which the integrand of the Powheg Sudakov form factor exponent can depart from its soft/collinear approximation. This option avoids potentially pathological situations wherein the Born term in the denominator of the PowheG Sudakov exponent enters a region of phase space in which it, itself, is vanishing faster than the real cross section in the numerator, when this approaches its soft/collinear factorized form. This vanishing of tree-level matrix elements is well known to occur in the context of, e.g., charged current weak interactions, in which scattering processes can 'switch-off' as certain kinematical configurations are approached, due to conflicting chirality and angular momentum constraints. ${ }^{10}$ In contrast to the WW case, in the WWJ-MinLO generator, the effects of this setting are generally negligible in all distributions which are not sensitive to high transverse momentum emission of the second hardest radiated parton in the event ( $\gtrsim 200 \mathrm{GeV}$ ). Moreover, even in such cases, where the predictions should be considered as simply LO accurate, the effect of the switch is modest, ranging up to $25 \%$ in the worst case considered here $\left(p_{\mathrm{T}, \mathrm{j}_{2}} \sim 500 \mathrm{GeV}\right)$.

The central renormalization and factorization scale choice for the WWJ-MinLO results is dictated by the MINLO' formalism [57]. The scale choice used for the strong coupling inside the integrand of the Sudakov form factor exponent is the conventional setting shown in eq. (2.4). All other instances of the strong coupling are evaluated at a scale given by the transverse momentum of the weak-boson pair. The factorization scale at which the PDFs are evaluated is also set to this value. The resummation scale used in the WWJMinlo Sudakov was already given in section 2.3 as $Q=m_{W W}$. For further details on the technical implementation of the MINLO' procedure, in particular the subtleties surrounding renormalization and factorization scale variations, we refer the reader to refs. [57, 63]. In the WW generator, the central renormalization and factorization scale choice, for the computation of the $\bar{B}$ function, is set to the invariant mass of the $W^{+} W^{-}$system $m_{W W}$. In both generators, the scale uncertainty bands in our plots have been obtained by varying $\mu_{R}$ and $\mu_{F}$, independently, up and down by a factor of two around their central values, while

\footnotetext{
${ }^{10}$ Some such configurations are suggested in the context of $W^{+} W^{-}$production in appendix B of the Powheg WW generator paper [47].
} 
keeping $\frac{1}{2} \leq \mu_{R} / \mu_{F} \leq 2$. The variation of $Q$ is in principle straightforward in the MinLO' framework, however, in accounting for the $K_{R}$ dependence of the Sudakov form factor, we probe the same type of higher-order terms, beyond NLO accuracy [57].

Unless stated otherwise, all figures show comparisons of the WW and WWJ-MinLO simulations, and are arranged pairwise. In each case the left- (right-) hand plot shows the perturbative uncertainty of the WW (WWJ-MinLO) calculation, and in the lower panel the ratio to the WW (WWJ-MiNLO) central result.

\subsubsection{Total cross sections}

Before launching into comparisons of differential distributions it will help us first to understand the total inclusive cross sections of the WW and WWJ-MinLo generators relative to one another, including their theoretical uncertainties. For the total inclusive $W^{+} W^{-}$ production cross sections we find that the predictions of MINLO' and the conventional WW NLO computation are in agreement to within about $4 \%$ obtaining $1.219_{-2.1 \%}^{+2.4 \%} \mathrm{pb}$ from the latter, and $1.174_{-4.8 \%}^{+7.2 \%} \mathrm{pb}$ from WWJ-MinLO. It is entirely natural that the two sets of results do not agree identically, since they differ at the level of explicit NNLO-sized terms relative to the leading order $W^{+} W^{-}$production process; WWJ-MINLO includes all ingredients but the two-loop virtual corrections to the full NNLO computation, for example. Thus, we regard the fact that the MINLO' and conventional NLO results agree at about the $4 \%$ level as being quite satisfactory - indeed the agreement is slightly better than that found previously for the case of $14 \mathrm{TeV}$ LHC Drell-Yan production in ref. [57].

We note that the WWJ-MINLO prediction comes with a theoretical uncertainty (due to scale variations) which is about a factor 2-3 larger than that from the WW generator. ${ }^{11}$ This feature has already been observed in the first MINLO' work concerning the Drell-Yan process [57], and subsequently in applying the MINLO' method to $H W / H Z$ production [82]; in both cases a similar-sized enlargement of the MINLO' scale uncertainty was found with respect to the corresponding conventional total inclusive NLO predictions.

As in ref. [57], we point out that, just as one can expect the central values of the predictions to differ, on account of intrinsic differences at the NNLO level, one should not be surprised to find similar-sized inequalities in the associated scale variations. Furthermore, it is well known that scale variations often don't give a reasonable estimate of the size of missing higher-order contributions at LO, or even NLO. For example, in $W^{+} W^{-}$production at LO there is zero renormalization scale dependence, since there is zero dependence on the strong coupling constant, thus the true theoretical uncertainty at that order is significantly underestimated. For what concerns our NLO results here though, there is very clear supporting evidence from the NNLO studies of $W^{+} W^{-}$production in ref. [30] that the scale uncertainties predicted by our WWJ-MINLO program are actually more reasonable, whereas those from conventional NLO substantially underestimate the true error.

\footnotetext{
${ }^{11}$ For the total inclusive cross section, the predictions of the WW generator are identical to those of conventional NLO, owing to the exact unitarity of NLOPS algorithms.
} 

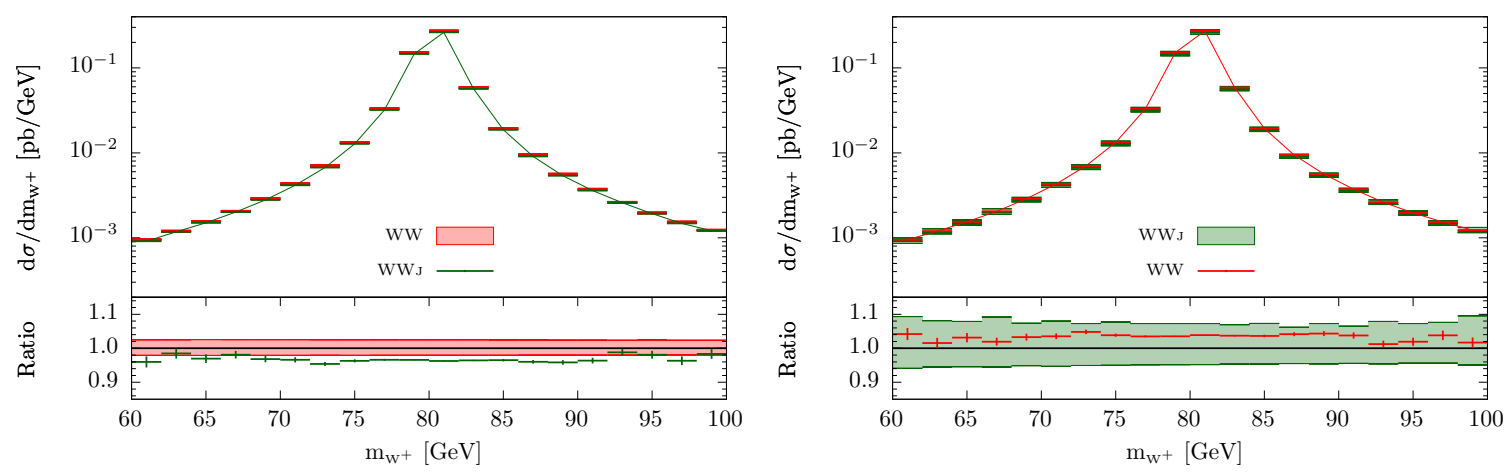

Figure 1. The mass of the $\mathrm{W}^{+}$boson as predicted by the WW (red) and WWJ-MinLo (dark green) generators. All results include parton shower and hadronization corrections obtained by processing the Powheg-Box hardest emission events with PyтнiA 8; MPI effects are not been included.

\subsubsection{Inclusive observables}

The preceding observations on the WW and WWJ-MINLO total inclusive cross sections are important to bear in mind while going on to examine kinematic distributions, since these similarities and differences are felt directly and indirectly by many of the plotted quantities. Indeed, for inclusive quantities, and to some extent also more exclusive ones, differences in normalization and uncertainty estimates are directly attributable to those found for the total inclusive cross sections.

We consider first differential distributions for observables which are inclusive with respect to the presence of QCD radiation. We begin in figure 1 with the $W^{+}$mass distribution. As expected, we see that the two generators agree rather well for this very inclusive quantity. The only differences between the two types of predictions here are to do with their normalization and the width of their scale uncertainty bands. As expected, these differences completely reflect those seen in the corresponding total inclusive cross sections discussed in section 3.1.1; the WWJ-MinLO result sits at the lower edge of the PowheG WW uncertainty band, which lies well within the larger WWJ-MinLO uncertainty band.

Next we show in figure 2 the transverse momentum of the $W^{+} W^{-}$system. At fixed order, predictions for this observable become divergent as $\mathrm{p}_{\mathrm{T}, \mathrm{W}^{+} \mathrm{w}^{-}}$approaches zero. All results shown here instead show a physical Sudakov peak and damping at small transverse momenta, owing to their all-orders resummation of soft/collinear emission effects; primarily via the Powheg Sudakov form factor in the case of the WW generator (red), and through the Minlo' Sudakov form factor and scale assignments in WWJ-Minlo without (blue) or with parton shower effects (green). In contrast to figure 1, the differences between the predictions of the WW (red) and WWJ-MinLO (green) generators here are not flat as a function of $\mathrm{p}_{\mathrm{T}, \mathrm{W}^{+} \mathrm{W}^{-}}$, and they are larger in magnitude, both at high and at low transverse momentum. The pure NLO level WWJ-MinLO predictions (blue) are shown here for the following discussion. However, we note that, below the Sudakov peak, this prediction is at best LL accurate for $\mathrm{p}_{\mathrm{T}, \mathrm{W}^{+} \mathrm{W}^{-}}$, since the argument of the form factor is directly the transverse momentum of the diboson system. 

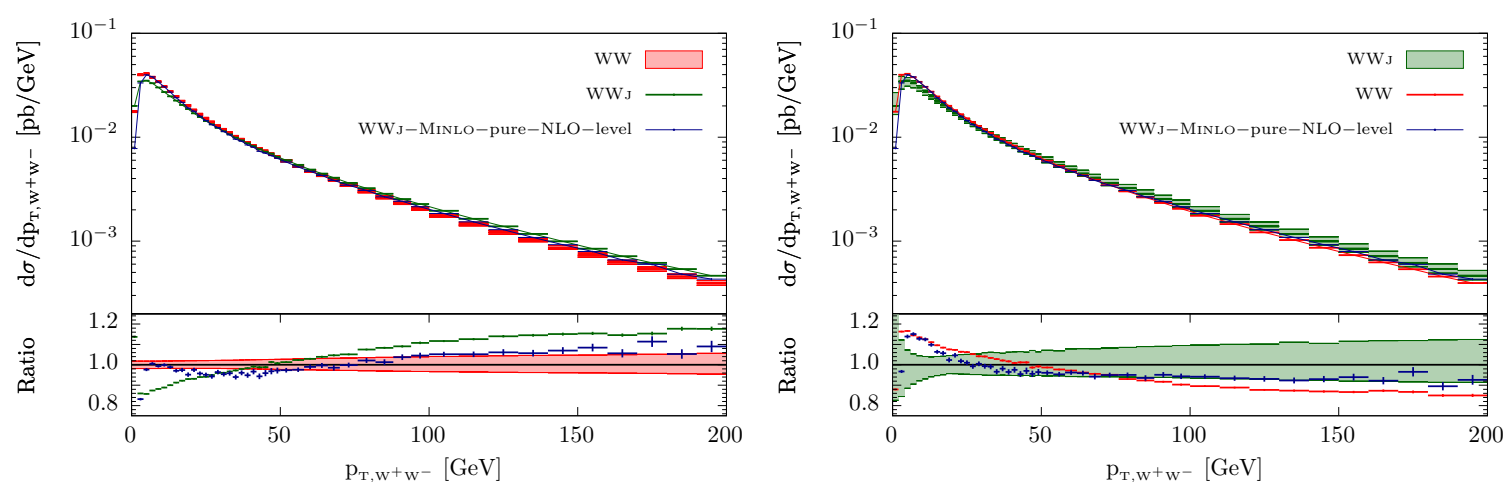

Figure 2. Transverse momentum of the $W^{+} W^{-}$system as predicted by the WW (red), WWJMinlo (dark green) generators and WWJ-Minlo at pure NLO level (blue). The simulation of parton shower and hadronization effects (but not MPI) is included with PYTHIA 8.

At high transverse momenta $\left(\mathrm{p}_{\mathrm{T}, \mathrm{W}^{+} \mathrm{W}^{-}} \sim 100 \mathrm{GeV}\right)$, only the WWJ-MinLO description is NLO accurate. In this region we observe that the WWJ-MINLO spectrum is harder than that of the WW generator and we interpret the modest $\sim 15 \%$ difference as being predominantly due to genuine NLO QCD corrections to the $\mathrm{p}_{\mathrm{T}, \mathrm{W}^{+} \mathrm{W}^{-}}$spectrum. In the low transverse momentum region the WW and WWJ-MinLO generators are seen to deviate from one another by up to $15 \%$ in the Sudakov peak region. On one hand we might well anticipate differences of roughly this size based on the fact that the WW and WWJMinLO generators should be expected to differ at roughly the level of NLL and $\mathrm{N}^{3} \mathrm{LL}_{\sigma}$ terms. On the other hand, naively at least, it is difficult to reconcile this large difference in the peak region with the fact that the two codes agree on the total inclusive cross section to within $3 \%$. Further investigation shows that in fact the bare NLO WWJ-MinLO $\mathrm{p}_{\mathrm{T}, \mathrm{w}^{+} \mathrm{w}^{-}}$ spectrum (blue line) actually follows that of the POWHEG WW generator remarkably well in the Sudakov peak region, with the two agreeing to better than $4-5 \%$ across in the region $\mathrm{p}_{\mathrm{T}, \mathrm{w}^{+} \mathrm{W}^{-}}<60 \mathrm{GeV}$. Noting this fact it becomes much easier to understand how the total inclusive cross sections can be in such good agreement, despite the apparently sizable differences in the Sudakov peak region. Essentially, the prediction of the bare WWJ-MinLO is subsequently acted on in the simulation chain, first by the POWHEG hardest radiation generator (generating the second hardest radiated parton in the case of WWJ-MINLO), and then by the parton shower. Both of these operations exactly respect the unitarity of the cross section, neither creating, deleting, or reweighting events, however, they will act to redistribute that cross section through the phase space, albeit consistently with NLO accuracy. Here, in particular, in the low $\mathrm{p}_{\mathrm{T}, \mathrm{w}^{+} \mathrm{W}^{-}}$region these multiple emission corrections have the effect of 'smearing out' the more peaked distribution from the bare NLO WWJMiNLO calculation (which, again, tracks closely that of WW), yielding the more blunt peak of the WWJ-Minlo generator seen in the plots. As a final remark on this aspect, we note also that these deviations at low $\mathrm{p}_{\mathrm{T}, \mathrm{w}^{+} \mathrm{W}^{-}}$correlate closely with similar ones in the transverse momentum spectra of the individual $W$ bosons themselves, for obvious reasons. We observe that the scale-uncertainty band of the WW code is once again smaller than the corresponding uncertainty for the WWJ-MINLO code. As discussed above, the latter code 

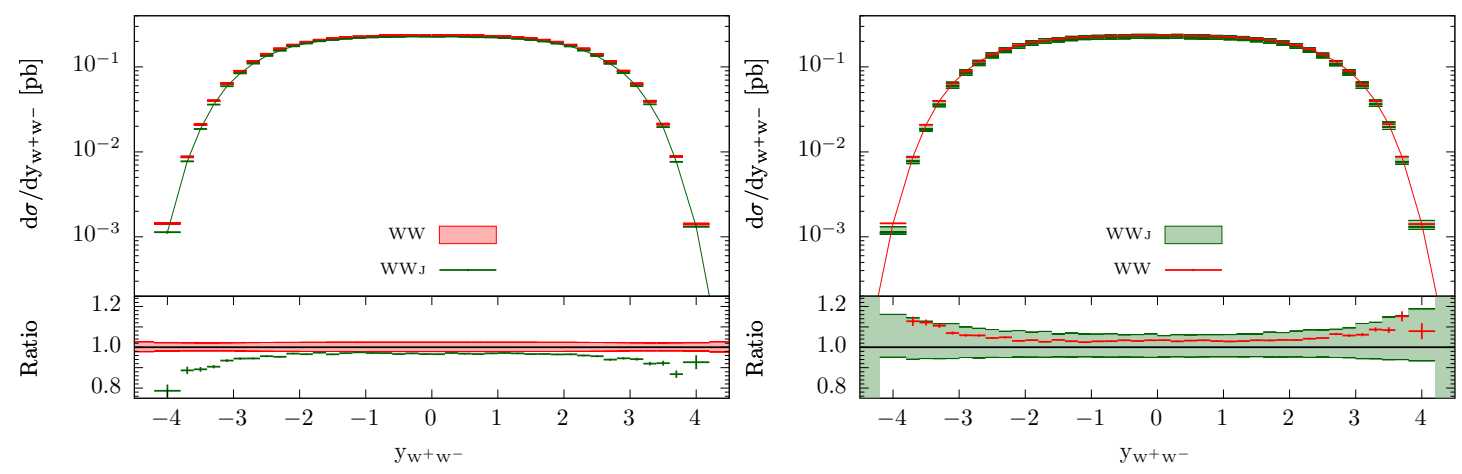

Figure 3. Rapidity of the $W^{+} W^{-}$system as predicted by the WW (red) and WWJ-MinLO (dark green) generators.

gives a more reliable estimate of the size of higher-order corrections which are not accounted for in our prediction. Remarkably similar trends to those shown here can be seen in the comparisons of the Powheg $\mathrm{W}$ and Z codes to WJ-Minlo and ZJ-Minlo respectively, for the $W$ and $Z p_{\text {T }}$ spectra, in ref. [57].

In figure 3 we show the rapidity of the $W^{+} W^{-}$pair. As in the case of the $W^{+}$mass distribution (figure 1), the WW central prediction lies within the uncertainty of the WWJMinlo generator. On the other hand, at high rapidities the WWJ-Minlo predictions are lower than the WW ones. Here again, the pattern of differences is quantitatively similar to that found in comparing $\mathrm{Z}$ and ZJ-MinLO predictions, for the $Z$ rapidity spectrum in the MINLO' implementations of ref. [57]. We add that the high-rapidity regions here, proportionally, contain more low $\mathrm{p}_{\mathrm{T}, \mathrm{W}^{+} \mathrm{W}^{-}}$events than the central domain. Thus, we suggest that the deviations seen at high rapidities, between the WW and WWJ-MinLO predictions, are strongly correlated with the comparable deviations in the $\mathrm{p}_{\mathrm{T}, \mathrm{W}^{+} \mathrm{W}^{-}}$spectrum of figure 2 . Similar behaviour is found for the rapidity distributions of the individual $W^{ \pm}$bosons, as well as their decay products.

The missing transverse momentum $\left(\mathrm{p}_{\mathrm{T}, \mathrm{miss}}\right)$ distribution is shown in figure 4 . Not surprisingly, this observable shows a pattern qualitatively similar to the one observed in figure 2 for the transverse momentum of the $W^{+} W^{-}$system. Although the $W^{+} W^{-}$transverse momentum is shared between leptons and neutrinos, one expects that the large $\mathrm{p}_{\mathrm{T}, \mathrm{miss}}$ tail is mostly populated by events where the $W^{+} W^{-}$system had a large boost, hence the WWJ-MinLO result displays a cross section larger than the WW one. The large differences observed at small values of $\mathrm{p}_{\mathrm{T}, \mathrm{W}^{+} \mathrm{W}^{-}}$get instead partly diluted when looking at $\mathrm{p}_{\mathrm{T}, \mathrm{miss}}$. This can be understood by considering the underlying weak decay $W^{+} W^{-} \rightarrow l^{+} l^{-} \nu \bar{\nu}$ : even when the $W^{+} W^{-}$system is almost at rest, the transverse momentum of each neutrino is of order $m_{W} / 2$ or more (depending on how boosted the $W$ boson is off which it is emitted). This consideration, together with the fact that the missing energy is the absolute value of a vectorial sum of two transverse momenta, justifies why differences that at the peak of the $\mathrm{p}_{\mathrm{T}, \mathrm{W}^{+} \mathrm{W}^{-}}$distribution reached a factor 1.16 become averaged out in the $\mathrm{p}_{\mathrm{T}, \mathrm{miss}}$ spectrum, which, at low values, only exhibits $10 \%$ differences at most between the WW and WWJ-MinLo simulations. 

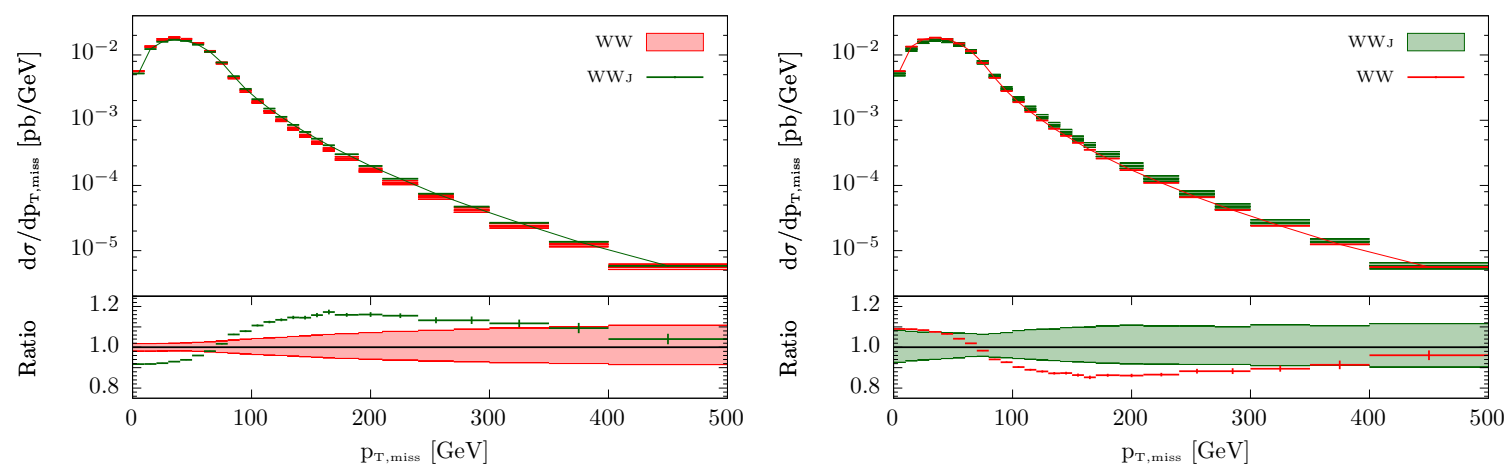

Figure 4. Missing transverse momentum as predicted by the WW (red) and WWJ-MinLO (dark green) generators.
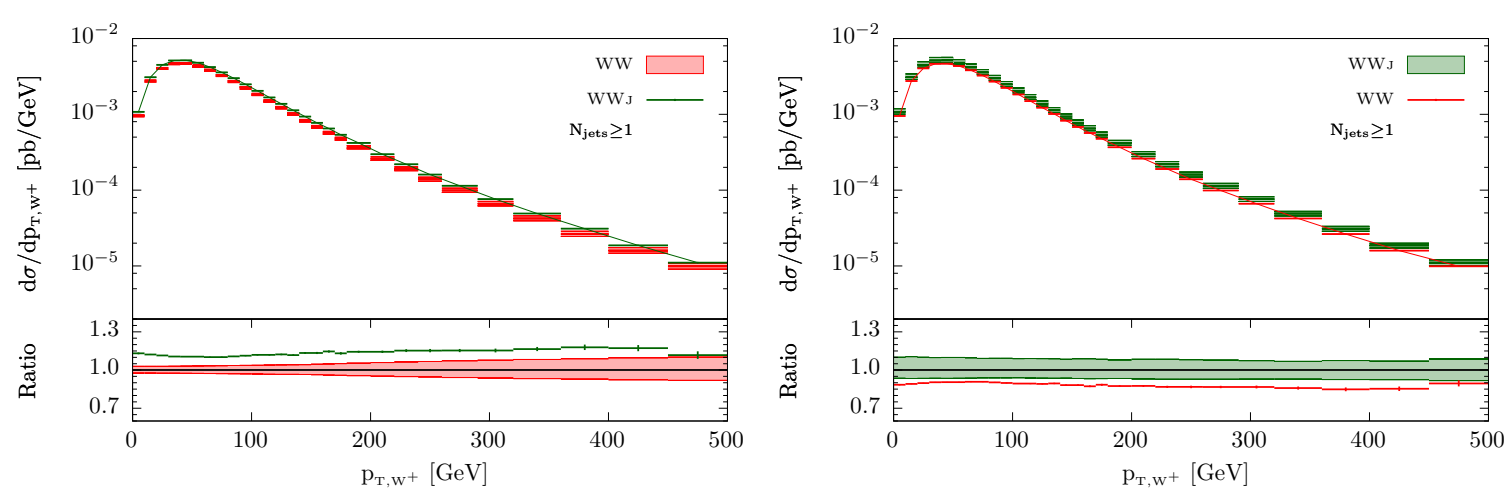

Figure 5. The $W^{+}$boson transverse momentum in the 1-jet region, as predicted by the WW (red) and WWJ-MinLO (dark green) generators.

\subsubsection{Jet associated production}

We now turn to results where at least one jet is required in the final state. Jets are reconstructed using the anti- $k_{t}$ algorithm [83] as implemented in FastJet [84]. In the following, we have chosen $R=0.4$ and jets are required to have $\mathrm{p}_{\mathrm{T}_{, \mathrm{j}_{1}}}>\mathrm{p}_{\mathrm{T}, \min }=25 \mathrm{GeV}$.

In figure 5 we show the $W^{+}$transverse momentum distribution in events with at least one jet. This distribution is described at NLO accuracy by both generators when no jet-cut is imposed. However, when we require to have one jet in the final state, the WW generator is only $\mathrm{LO}$ accurate. In this case, due to the inclusive nature of this observable with respect to extra QCD radiation, NLO corrections amount to an overall $K$ factor, and hence we find good agreement in the shape of the two distributions.

In figure 6 we now examine the transverse momentum distribution of the $W^{+} W^{-}$ system in events with at least one jet. In this case, when the transverse momentum is large $\left(\mathrm{p}_{\mathrm{T}, \mathrm{W}^{+} \mathrm{W}^{-}} \gg \mathrm{p}_{\mathrm{T}, \min }\right)$ we recover the behaviour observed in figure 2 . On the other hand we observe a very different behaviour at small $\mathrm{p}_{\mathrm{T}, \mathrm{w}^{+} \mathrm{w}^{-}}$. Given the jet requirement, the region $0<\mathrm{p}_{\mathrm{T}, \mathrm{W}^{+} \mathrm{W}^{-}}<25 \mathrm{GeV}$ is populated only by events with at least two $\mathrm{QCD}$ emissions. Therefore, for the WW generator this region is populated by the parton shower only. For the WWJ-Minlo generator, on the other hand, two-parton configurations are 

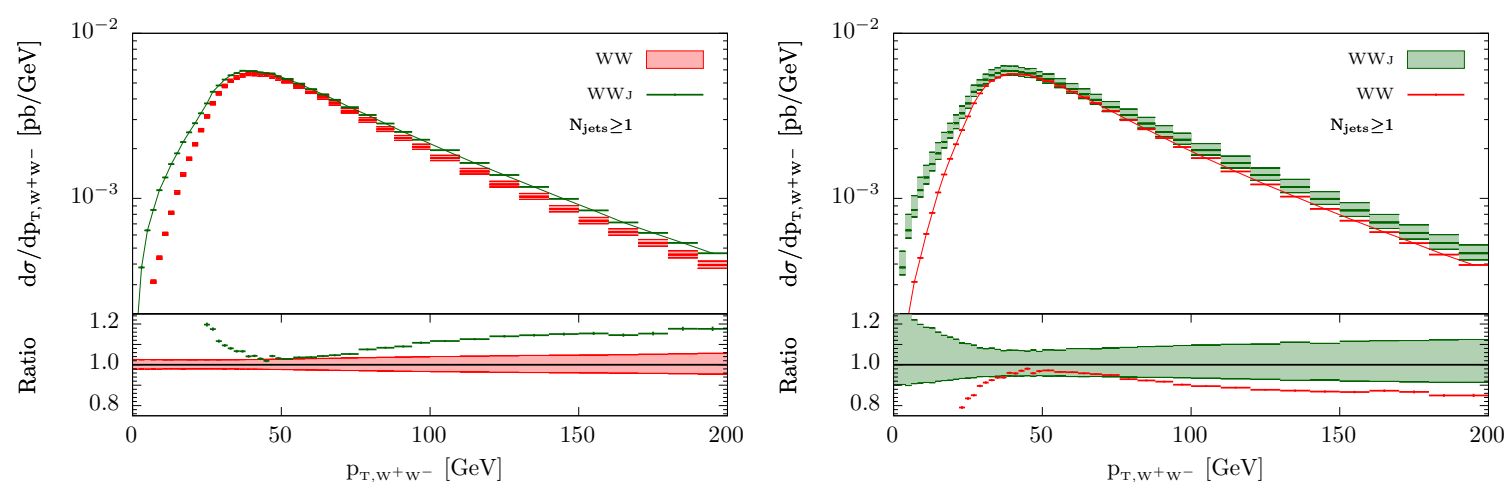

Figure 6. The $W^{+} W^{-}$transverse momentum in the 1-jet region, as predicted by the WW (red) and WWJ-MinLo (dark green) generators.

also provided by leading order matrix elements; these tend to populate this region more strongly than the shower alone since they tend to provide harder QCD radiation against which the first jet can recoil, leaving room for $\mathrm{p}_{\mathrm{T}, \mathrm{W}^{+} \mathrm{W}^{-}}$to be smaller. The shape change that we observe for this distribution at $\mathrm{p}_{\mathrm{T}, \mathrm{W}^{+} \mathrm{W}^{-}}=25 \mathrm{GeV}$ has to do with the WWJ-MinLO code switching from being $\mathrm{LO}$ accurate below that threshold to NLO accurate above it. The LO behaviour of the WWJ-MINLO generator in this region is also evident from the widening of the WWJ-MinLO uncertainty band below $\mathrm{p}_{\mathrm{T}, \mathrm{w}^{+} \mathrm{W}^{-}}=25 \mathrm{GeV}$.

In figure 7 we show the transverse momentum spectrum of the hardest jet. ${ }^{12}$ This is a quantity that is described only at LO level by the WW generator, whereas NLO corrections are included in the WWJ-MinLO program. We notice that the two results start to deviate at fairly modest transverse momenta, and differences up to a factor of almost 2 can be noticed in the tail. This is due to the absence of radiative corrections in the WW generator. In particular we have verified that in the tail region the contribution from events containing 2 well separated partons is sizable. The WW program underestimates their rates (as shown also in the next plot), since it doesn't contain the corresponding exact matrix elements. The uncertainty band of the WWJ-MinLo result shows the size and pattern that one would expect to see in a NLO-accurate prediction. On the other hand, especially in the low-to-medium range, the uncertainty band for the WW result is thinner than the WWJ-MINLO one, despite the nominal accuracy of the WW generator is just LO for this observable. This is explained as follows: in this region the scale variation for the WW predictions is due to the scale dependence of the $\bar{B}$ function, which is a NLO-accurate quantity. At larger $\mathrm{p}_{\mathrm{T}}$ values, where bornzerodamp is active, the band slowly thickens, giving an uncertainty of $\pm 10 \%$.

Another interesting observable to consider is the transverse momentum spectrum of the second hardest jet, which we show in figure 8. Not surprisingly, here we observe huge differences among the two generators. In the WW code only the hardest radiation is generated by Powheg. Hence the particles that constitute the second jet are only produced

\footnotetext{
${ }^{12}$ The relatively low value of the cross section in the first non-zero bin is a histogram edge effect: the first non-zero histogram bin goes from 20 to $30 \mathrm{GeV}$, with the $\mathrm{p}_{\mathrm{T}, \mathrm{j}_{1}}$ cut being at $25 \mathrm{GeV}$.
} 

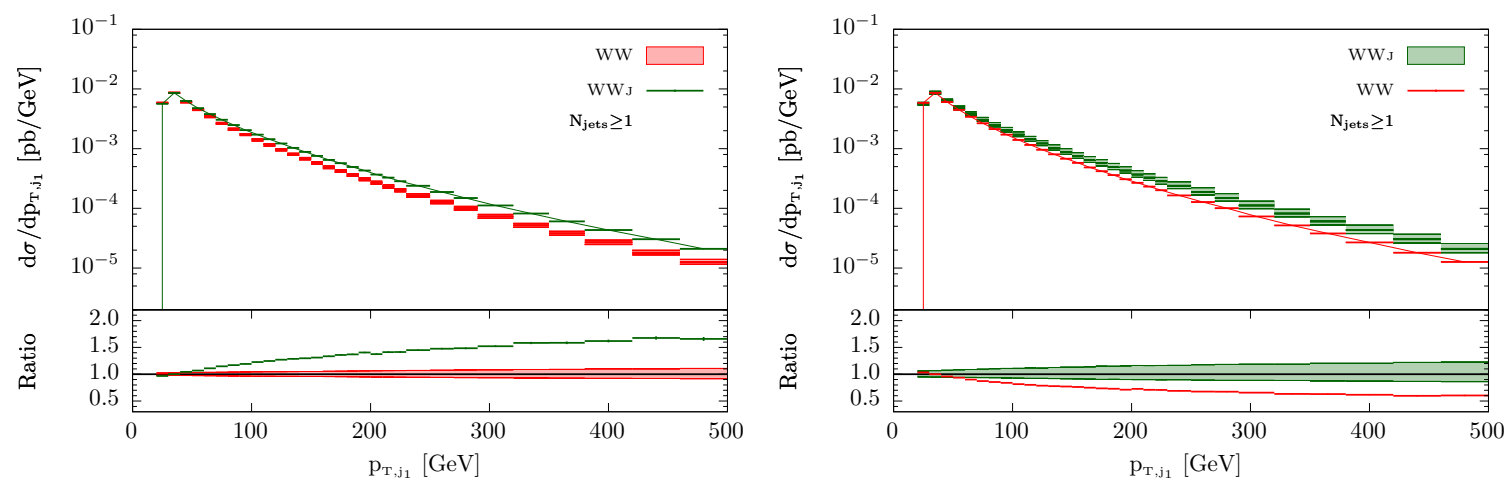

Figure 7. Leading jet transverse momentum as predicted by the WW (red) and WWJ-MinLO (dark green) generators.
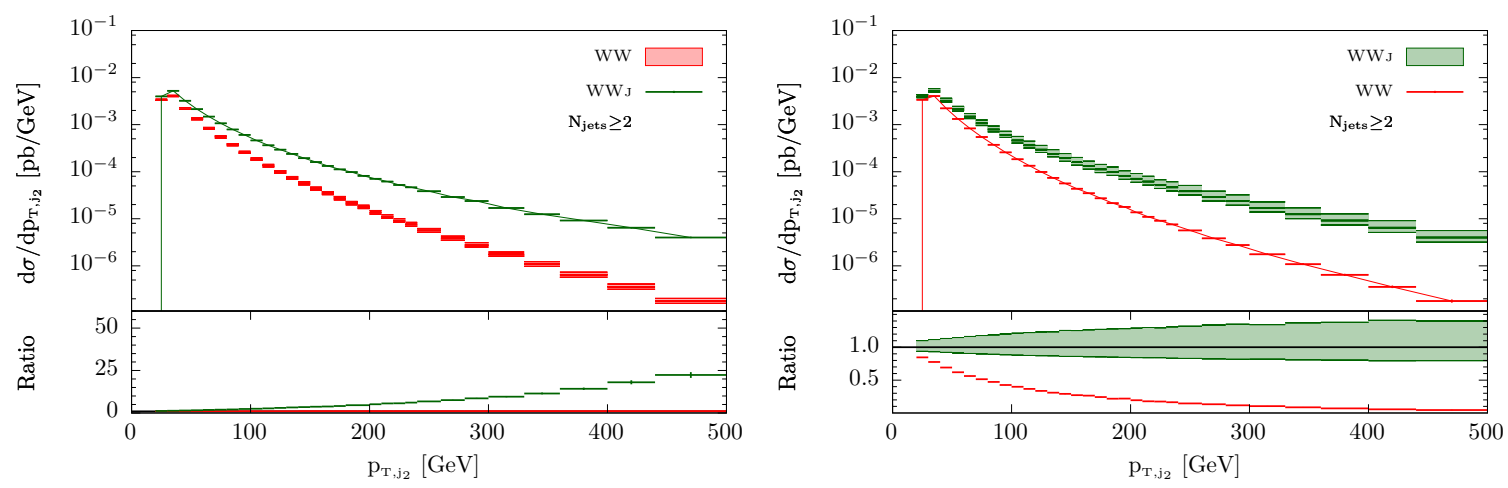

Figure 8. Second jet transverse momentum as predicted by the WW (red) and WWJ-MinLO (dark green) generators.

via parton showering: when large $\mathrm{p}_{\mathrm{T}, \mathrm{j}_{2}}$ regions are probed, the $\mathrm{WW}$ code is bound to predict an unreliable cross section (too small in this case). The WWJ-MINLO prediction is instead more accurate, since the matrix elements describing the production of two separated outgoing partons are included exactly, although only at LO. The LO nature of this result is reflected in the relatively large uncertainty band.

After having shown how the WWJ-MINLO generator compares against the WW one for jet observables, we find it useful to compare, for the same observables, WWJ-MinLO against a NLO computation (without any Minlo improvement) for the process $p p \rightarrow W^{+} W^{-} j{ }^{13}$ These comparisons are shown in figure 9. In the left panel we observe that the WWJ-MinLO prediction for the hardest jet $\mathrm{p}_{\mathrm{T}}$ spectrum agrees very well with the fixed-order result for the transverse momentum spectrum of the hardest jet. The moderate differences close to the threshold to produce one jet are likely due to the use of different scales as well as to the presence of the Minlo Sudakov. At larger values of $\mathrm{p}_{\mathrm{T}, \mathrm{j}_{1}}$ the two predictions are compatible, although the NLO result exhibits a slightly harder spectrum. This is easily explained by

\footnotetext{
${ }^{13}$ This result was obtained running at fixed-order the WWJ-MinLo code, switching off the MinLO machinery but including a $10 \mathrm{GeV}$ generation cut for the hardest parton transverse momentum. Renormalization and factorization scales have been set equal to $2 m_{W}$.
} 

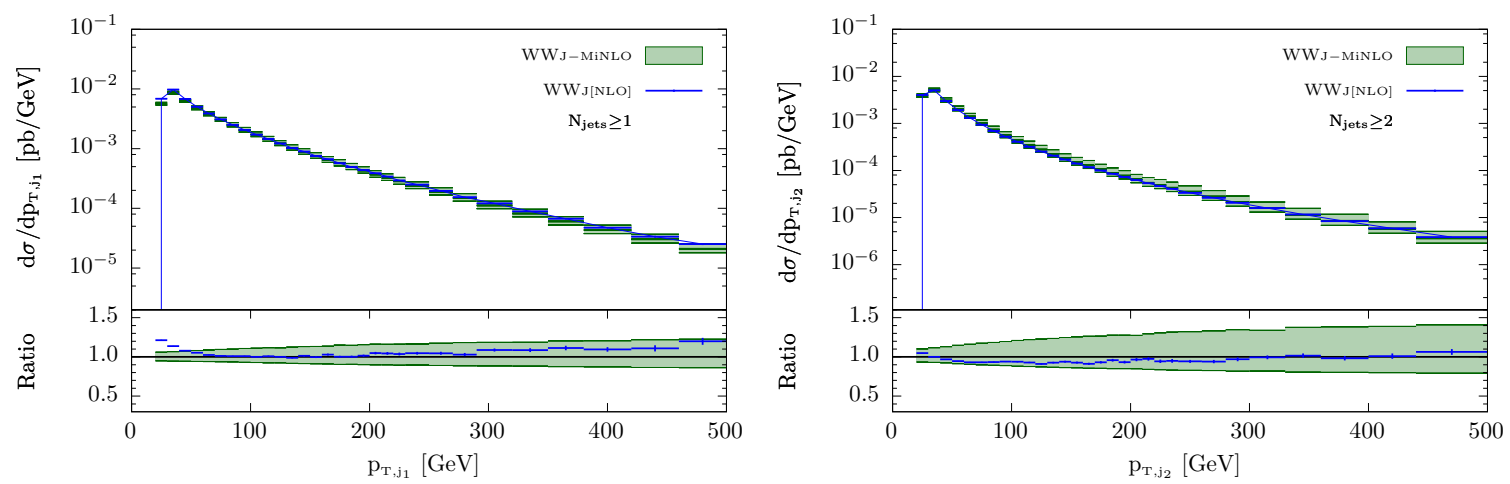

Figure 9. First and second jet transverse momentum as predicted by the WWJ-MinLO (dark green) generators compared against a fixed-order NLO computation for the same process (WWJ [NLO], blue).

recalling that, at large transverse momenta, although the Minlo Sudakov switches off, the WWJ-Minlo scale prescription is used: the WWJ-Minlo line is obtained with a dynamical scale choice, i.e. a scale choice certainly larger than the one used for the NLO computation, where we have chosen $\mu=2 m_{W}$. This consideration is also supported by the fact that the NLO and WWJ-MinLO predictions agree extremely well for $\mathrm{p}_{\mathrm{T}, \mathrm{j}_{1}} \simeq 2 m_{W}$. In the right panel of figure 9 we show instead the comparison for the second hardest jet, which agree quite well.

\section{Conclusion and outlook}

In this paper we have extended the MinLO' method to merge $W^{+} W^{-}$and $W^{+} W^{-}+$jet NLO calculations, preserving NLO accuracy throughout the 0 - and 1-jet phase space. The merging is achieved without the introduction of an unphysical merging scale; such a scale formally spoils the NLO accuracy of merged samples in some regions of phase space.

The work presented here exemplifies the extension of the MINLO' method, in its original form, to general colour singlet production processes, where the virtual corrections to the lowest multiplicity process are non-trivial. The method relies on the fact that the NNLL transverse momentum resummation coefficient $B_{2}$ is the same in $W^{+} W^{-}$and Drell-Yan production, save for a process-dependent term proportional to the virtual corrections affecting the leading order process. By carefully replacing this process-dependent component in the MiNLO' recipe for Drell-Yan type reactions, with the analogous virtual correction to $W^{+} W^{-}$ production, the MinLO' method becomes directly applicable to the latter. The same replacement procedure holds for extending MINLO' to all colour singlet production processes.

We have performed an extensive phenomenological study, comparing our results to conventional $W^{+} W^{-}$NLOPS and $W^{+} W^{-}+$jet NLO calculations. In general we find good agreement, with standard NLO results lying within the uncertainty band of the MINLO' improved prediction. On the other hand, the scale uncertainty of the MinLO' results for inclusive observables, while being less than $7 \%$, is larger than that of conventional NLO, by a factor of $2-3$. 
Although it has been proven that the MINLO' method yields NLO accuracy for 0-jet and inclusive quantities, and that scale variations therein give rise to only NNLO-sized shifts, the precise value of these relative $\mathcal{O}\left(\alpha_{\mathrm{S}}^{2}\right)$ ambiguities is understood to be formally different in each case. The propagation of scale variation in the MinLO' approach is complicated, however, it's somewhat natural to expect that MINLO' should tend to exhibit a larger scale uncertainty than conventional NLO calculations: in the former, the scale for evaluating the strong coupling constant and PDFs is mandatorily $\mathrm{p}_{\mathrm{T}, \mathrm{W}^{+} \mathrm{W}^{-}}$, thus, scale variation for the great majority of events forming the inclusive cross section, in the vicinity of the Sudakov peak, takes place around significantly smaller values in MINLO' than in conventional NLO computations $\left(\mu_{R} \sim \mu_{F} \gtrsim \mathcal{O}\left(m_{W}\right)\right)$. Considering the differences reported between NLO and NNLO results in the recent study of ref. [30], we regard the theoretical uncertainty estimate from our MiNLO' computation as being quite reasonable, while that of conventional NLO is a poor underestimate.

In conclusion we find that the WWJ-MinLO generator supersedes the WW generator, retaining the NLO accuracy of the latter for inclusive quantities and augmenting it with a more reliable, NLO accurate description of hard radiation. Furthermore, for fully inclusive, 0 -jet, and 1-jet quantities the WWJ-MINLO generator gives a more realistic estimate of the theoretical uncertainty.

Finally, we remark that with a MINLO' accurate simulation at hand, it becomes straightforward, theoretically, to upgrade it, through a reweighting procedure, to obtain NNLOPS accurate predictions. In the present case, taking into account the $W^{+} W^{-}$decays, the high dimensionality of the Born phase space makes this task far from trivial in practice. We leave this to future work.

\section{Acknowledgments}

We would like to thank G. Luisoni for his assistance with the code GoSAM. The research of GZ is supported by the ERC consolidator grant 614577 HICCUP - High Impact Cross section Calculations for Ultimate Precision. PM and ER have also benefited from the ERC grant HICCUP. TM is supported by U.S. DOE grant DE-AC02-05CH11231 and acknowledges computational resources provided through ERC grant number 291377 LHCtheory. PM was partly supported by the Swiss National Science Foundation (SNF) under grant PBZHP2-147297. We all gratefully acknowledge the Mainz Institute for Theoretical Physics (MITP) for its hospitality and support while part of this work was carried out. Additionally, GZ gratefully acknowledges KITP and PM and ER acknowledge the CERN's Theory Department for hospitality while completing this work.

Open Access. This article is distributed under the terms of the Creative Commons Attribution License (CC-BY 4.0), which permits any use, distribution and reproduction in any medium, provided the original author(s) and source are credited. 


\section{References}

[1] CDF collaboration, T. Aaltonen et al., Measurement of the $W^{+} W^{-}$Production Cross section and Search for Anomalous $W W \gamma$ and $W W Z$ Couplings in $p \bar{p}$ Collisions at $\sqrt{s}=1.96$ TeV, Phys. Rev. Lett. 104 (2010) 201801 [arXiv:0912.4500] [INSPIRE].

[2] CDF collaboration, T. Aaltonen et al., Measurement of the $W^{+} W^{-}$Production Cross section and Differential Cross section with Jets in $p \bar{p}$ Collisions at $\sqrt{s}=1.96$ TeV, http://www-cdf.fnal.gov/physics/ewk/2014/WWjets/cdf11098_WW+jets.pdf.

[3] D0 collaboration, V.M. Abazov et al., Limits on anomalous trilinear gauge boson couplings from $W W, W Z$ and $W \gamma$ production in $p \bar{p}$ collisions at $\sqrt{s}=1.96$ TeV, Phys. Lett. B 718 (2012) 451 [arXiv: 1208.5458] [INSPIRE].

[4] ATLAS collaboration, Measurement of total and differential $W^{+} W^{-}$production cross sections in proton-proton collisions at $\sqrt{s}=8 \mathrm{TeV}$ with the ATLAS detector and limits on anomalous triple-gauge-boson couplings, arXiv:1603.01702 [INSPIRE].

[5] CMS collaboration, Measurement of the $W^{+} W^{-}$cross section in pp collisions at $\sqrt{s}=$ $8 \mathrm{TeV}$ and limits on anomalous gauge couplings, Eur. Phys. J. C 76 (2016) 401 [arXiv: 1507.03268] [INSPIRE].

[6] R.W. Brown and K.O. Mikaelian, $W^{+} W^{-}$and $Z^{0} Z^{0}$ Pair Production in $e^{+} e^{-}, p p, p \bar{p}$ Colliding Beams, Phys. Rev. D 19 (1979) 922 [inSPIRE].

[7] J. Ohnemus, An Order $\alpha^{-} s$ calculation of hadronic $W^{-} W^{+}$production, Phys. Rev. D 44 (1991) 1403 [INSPIRE].

[8] S. Frixione, A Next-to-leading order calculation of the cross-section for the production of $W^{+} W^{-}$pairs in hadronic collisions, Nucl. Phys. B 410 (1993) 280 [INSPIRE].

[9] L.J. Dixon, Z. Kunszt and A. Signer, Helicity amplitudes for $O\left(\alpha_{s}\right)$ production of $W^{+} W^{-}$, $W^{ \pm} Z, Z Z, W^{ \pm} \gamma$, or $Z \gamma$ pairs at hadron colliders, Nucl. Phys. B 531 (1998) 3 [hep-ph/9803250] [INSPIRE].

[10] J.M. Campbell and R.K. Ellis, An update on vector boson pair production at hadron colliders, Phys. Rev. D 60 (1999) 113006 [hep-ph/9905386] [INSPIRE].

[11] L.J. Dixon, Z. Kunszt and A. Signer, Vector boson pair production in hadronic collisions at order $\alpha_{s}$ : Lepton correlations and anomalous couplings, Phys. Rev. D 60 (1999) 114037 [hep-ph/9907305] [INSPIRE].

[12] J.M. Campbell, R.K. Ellis and C. Williams, Vector boson pair production at the LHC, JHEP 07 (2011) 018 [arXiv: 1105.0020] [INSPIRE].

[13] S. Dittmaier, S. Kallweit and P. Uwer, NLO QCD corrections to $W W+$ jet production at hadron colliders, Phys. Rev. Lett. 100 (2008) 062003 [arXiv:0710.1577] [INSPIRE].

[14] J.M. Campbell, R.K. Ellis and G. Zanderighi, Next-to-leading order predictions for $W W+1$ jet distributions at the LHC, JHEP 12 (2007) 056 [arXiv:0710.1832] [INSPIRE].

[15] S. Dittmaier, S. Kallweit and P. Uwer, NLO QCD corrections to $p p / p \bar{p} \rightarrow W W+j e t+X$ including leptonic W-boson decays, Nucl. Phys. B 826 (2010) 18 [arXiv:0908.4124] [INSPIRE].

[16] T. Melia, K. Melnikov, R. Rontsch and G. Zanderighi, NLO QCD corrections for $W^{+} W^{-}$ pair production in association with two jets at hadron colliders, Phys. Rev. D 83 (2011) 114043 [arXiv:1104.2327] [INSPIRE]. 
[17] D.A. Dicus, C. Kao and W.W. Repko, Gluon Production of Gauge Bosons, Phys. Rev. D 36 (1987) 1570 [INSPIRE].

[18] E.W.N. Glover and J.J. van der Bij, Vector boson pair production via gluon fusion, Phys. Lett. B 219 (1989) 488 [inSPIRE].

[19] T. Binoth, M. Ciccolini, N. Kauer and M. Krämer, Gluon-induced WW background to Higgs boson searches at the LHC, JHEP 03 (2005) 065 [hep-ph/0503094] [INSPIRE].

[20] T. Binoth, M. Ciccolini, N. Kauer and M. Krämer, Gluon-induced W-boson pair production at the LHC, JHEP 12 (2006) 046 [hep-ph/0611170] [INSPIRE].

[21] J.M. Campbell, R.K. Ellis and C. Williams, Gluon-Gluon Contributions to $W^{+} W^{-}$ Production and Higgs Interference Effects, JHEP 10 (2011) 005 [arXiv:1107.5569] [INSPIRE].

[22] T. Melia, K. Melnikov, R. Rontsch, M. Schulze and G. Zanderighi, Gluon fusion contribution to $W^{+} W^{-}+$jet production, JHEP 08 (2012) 115 [arXiv:1205.6987] [INSPIRE].

[23] T. Gehrmann et al., $W^{+} W^{-}$Production at Hadron Colliders in Next to Next to Leading Order QCD, Phys. Rev. Lett. 113 (2014) 212001 [arXiv:1408. 5243] [inSPIRE].

[24] T. Gehrmann, A. von Manteuffel, L. Tancredi and E. Weihs, The two-loop master integrals for $q \bar{q} \rightarrow V V$, JHEP 06 (2014) 032 [arXiv: 1404.4853] [INSPIRE].

[25] F. Caola, J.M. Henn, K. Melnikov, A.V. Smirnov and V.A. Smirnov, Two-loop helicity amplitudes for the production of two off-shell electroweak bosons in quark-antiquark collisions, JHEP 11 (2014) 041 [arXiv:1408.6409] [INSPIRE].

[26] T. Gehrmann, A. von Manteuffel and L. Tancredi, The two-loop helicity amplitudes for $q \bar{q}^{\prime} \rightarrow V_{1} V_{2} \rightarrow 4$ leptons, JHEP 09 (2015) 128 [arXiv: 1503.04812] [INSPIRE].

[27] F. Caola, J.M. Henn, K. Melnikov, A.V. Smirnov and V.A. Smirnov, Two-loop helicity amplitudes for the production of two off-shell electroweak bosons in gluon fusion, JHEP 06 (2015) 129 [arXiv: 1503.08759] [INSPIRE].

[28] A. von Manteuffel and L. Tancredi, The two-loop helicity amplitudes for $g g \rightarrow V_{1} V_{2} \rightarrow 4$ leptons, JHEP 06 (2015) 197 [arXiv:1503.08835] [INSPIRE].

[29] F. Caola, K. Melnikov, R. Röntsch and L. Tancredi, QCD corrections to $W^{+} W^{-}$production through gluon fusion, Phys. Lett. B 754 (2016) 275 [arXiv:1511.08617] [InSPIRE].

[30] M. Grazzini, S. Kallweit, S. Pozzorini, D. Rathlev and M. Wiesemann, $W^{+} W^{-}$production at the LHC: fiducial cross sections and distributions in NNLO QCD, JHEP 08 (2016) 140 [arXiv: 1605. 02716] [INSPIRE].

[31] A. Bierweiler, T. Kasprzik, J.H. Kühn and S. Uccirati, Electroweak corrections to W-boson pair production at the LHC, JHEP 11 (2012) 093 [arXiv: 1208.3147] [INSPIRE].

[32] J. Baglio, L.D. Ninh and M.M. Weber, Massive gauge boson pair production at the LHC: a next-to-leading order story, Phys. Rev. D 88 (2013) 113005 [arXiv:1307.4331] [INSPIRE].

[33] M. Billóni, S. Dittmaier, B. Jäger and C. Speckner, Next-to-leading order electroweak corrections to $p p \rightarrow W^{+} W^{-} \rightarrow 4$ leptons at the LHC in double-pole approximation, JHEP 12 (2013) 043 [arXiv: 1310.1564] [InSPIRE].

[34] B. Biedermann et al., Next-to-leading-order electroweak corrections to $p p \rightarrow W^{+} W^{-} \rightarrow 4$ leptons at the LHC, JHEP 06 (2016) 065 [arXiv: 1605.03419] [INSPIRE]. 
[35] M. Grazzini, Soft-gluon effects in WW production at hadron colliders, JHEP 01 (2006) 095 [hep-ph/0510337] [INSPIRE].

[36] Y. Wang, C.S. Li, Z.L. Liu, D.Y. Shao and H.T. Li, Transverse-Momentum Resummation for Gauge Boson Pair Production at the Hadron Collider, Phys. Rev. D 88 (2013) 114017 [arXiv: 1307.7520] [INSPIRE].

[37] P. Meade, H. Ramani and M. Zeng, Transverse momentum resummation effects in $W^{+} W^{-}$ measurements, Phys. Rev. D 90 (2014) 114006 [arXiv:1407.4481] [INSPIRE].

[38] M. Grazzini, S. Kallweit, D. Rathlev and M. Wiesemann, Transverse-momentum resummation for vector-boson pair production at NNLL+NNLO, JHEP 08 (2015) 154 [arXiv: 1507.02565] [INSPIRE].

[39] S. Dawson, I.M. Lewis and M. Zeng, Threshold resummed and approximate next-to-next-to-leading order results for $W^{+} W^{-}$pair production at the LHC, Phys. Rev. D 88 (2013) 054028 [arXiv: 1307.3249] [INSPIRE].

[40] P. Jaiswal and T. Okui, Explanation of the $W W$ excess at the LHC by jet-veto resummation, Phys. Rev. D 90 (2014) 073009 [arXiv:1407.4537] [INSPIRE].

[41] T. Becher, R. Frederix, M. Neubert and L. Rothen, Automated NNLL + NLO resummation for jet-veto cross sections, Eur. Phys. J. C 75 (2015) 154 [arXiv:1412.8408] [inSPIRE].

[42] P.F. Monni and G. Zanderighi, On the excess in the inclusive $W^{+} W^{-} \rightarrow l^{+} l^{-} \nu \bar{\nu}$ cross section, JHEP 05 (2015) 013 [arXiv: 1410.4745] [INSPIRE].

[43] S. Dawson, P. Jaiswal, Y. Li, H. Ramani and M. Zeng, Resummation of Jet Veto Logarithms at partial $N^{3} L L+N N L O$ for $W^{+} W^{-}$Production at the LHC, Submitted to: Phys. Rev. D (2016) [arXiv: 1606. 01034] [INSPIRE].

[44] S. Frixione and B.R. Webber, Matching NLO QCD computations and parton shower simulations, JHEP 06 (2002) 029 [hep-ph/0204244] [INSPIRE].

[45] K. Hamilton, A positive-weight next-to-leading order simulation of weak boson pair production, JHEP 01 (2011) 009 [arXiv: 1009.5391] [INSPIRE].

[46] S. Höche, F. Krauss, M. Schonherr and F. Siegert, Automating the POWHEG method in Sherpa, JHEP 04 (2011) 024 [arXiv: 1008.5399] [inSPIRE].

[47] T. Melia, P. Nason, R. Rontsch and G. Zanderighi, $W+W$-, $W Z$ and $Z Z$ production in the POWHEG BOX, JHEP 11 (2011) 078 [arXiv:1107.5051] [InSPIRE].

[48] P. Nason and G. Zanderighi, $W^{+} W^{-}, W Z$ and $Z Z$ production in the POWHEG-BOX-V2, Eur. Phys. J. C 74 (2014) 2702 [arXiv:1311.1365] [InSPIRE].

[49] J. Bellm et al., Anomalous coupling, top-mass and parton-shower effects in $W^{+} W^{-}$ production, JHEP 05 (2016) 106 [arXiv: 1602.05141] [INSPIRE].

[50] J. Bellm et al., Herwig 7.0/Herwigt+ 3.0 release note, Eur. Phys. J. C 76 (2016) 196 [arXiv: 1512.01178] [INSPIRE].

[51] F. Cascioli, S. Höche, F. Krauss, P. Maierhöfer, S. Pozzorini and F. Siegert, Precise Higgs-background predictions: merging NLO QCD and squared quark-loop corrections to four-lepton + 0,1 jet production, JHEP 01 (2014) 046 [arXiv: 1309.0500] [INSPIRE].

[52] T. Gehrmann, S. Höche, F. Krauss, M. Schonherr and F. Siegert, NLO QCD matrix elements + parton showers in $e^{+} e^{-} \rightarrow$ hadrons, JHEP 01 (2013) 144 [arXiv:1207.5031] [INSPIRE]. 
[53] S. Hoeche, F. Krauss, M. Schonherr and F. Siegert, QCD matrix elements + parton showers: The NLO case, JHEP 04 (2013) 027 [arXiv:1207.5030] [INSPIRE].

[54] R. Frederix, S. Frixione, V. Hirschi, F. Maltoni, R. Pittau and P. Torrielli, Four-lepton production at hadron colliders: aMC@NLO predictions with theoretical uncertainties, JHEP 02 (2012) 099 [arXiv: 1110.4738] [INSPIRE].

[55] R. Frederix and S. Frixione, Merging meets matching in MC@NLO, JHEP 12 (2012) 061 [arXiv: 1209.6215] [INSPIRE].

[56] J. Alwall et al., The automated computation of tree-level and next-to-leading order differential cross sections and their matching to parton shower simulations, JHEP 07 (2014) 079 [arXiv: 1405.0301] [INSPIRE].

[57] K. Hamilton, P. Nason, C. Oleari and G. Zanderighi, Merging $H / W / Z+0$ and 1 jet at $N L O$ with no merging scale: a path to parton shower + NNLO matching, JHEP 05 (2013) 082 [arXiv: 1212.4504] [INSPIRE].

[58] N. Kauer and G. Passarino, Inadequacy of zero-width approximation for a light Higgs boson signal, JHEP 08 (2012) 116 [arXiv:1206.4803] [INSPIRE].

[59] P. Nason, A new method for combining NLO QCD with shower Monte Carlo algorithms, JHEP 11 (2004) 040 [hep-ph/0409146] [INSPIRE].

[60] S. Frixione, P. Nason and C. Oleari, Matching NLO QCD computations with Parton Shower simulations: the POWHEG method, JHEP 11 (2007) 070 [arXiv:0709.2092] [INSPIRE].

[61] S. Alioli, P. Nason, C. Oleari and E. Re, A general framework for implementing NLO calculations in shower Monte Carlo programs: the POWHEG BOX, JHEP 06 (2010) 043 [arXiv: 1002 .2581] [INSPIRE].

[62] W. Astill, W. Bizon, E. Re and G. Zanderighi, NNLOPS accurate associated HW production, JHEP 06 (2016) 154 [arXiv: 1603.01620] [InSPIRE].

[63] R. Frederix and K. Hamilton, Extending the MINLO method, JHEP 05 (2016) 042 [arXiv: 1512.02663] [INSPIRE].

[64] K. Hamilton, P. Nason, E. Re and G. Zanderighi, NNLOPS simulation of Higgs boson production, JHEP 10 (2013) 222 [arXiv: 1309.0017] [INSPIRE].

[65] K. Hamilton, P. Nason and G. Zanderighi, Finite quark-mass effects in the NNLOPS POWHEG+MiNLO Higgs generator, JHEP 05 (2015) 140 [arXiv:1501.04637] [INSPIRE].

[66] A. Karlberg, E. Re and G. Zanderighi, NNLOPS accurate Drell-Yan production, JHEP 09 (2014) 134 [arXiv: 1407.2940] [INSPIRE].

[67] S. Alioli et al., Combining Higher-Order Resummation with Multiple NLO Calculations and Parton Showers in GENEVA, JHEP 09 (2013) 120 [arXiv:1211.7049] [INSPIRE].

[68] S. Alioli, C.W. Bauer, C. Berggren, F.J. Tackmann, J.R. Walsh and S. Zuberi, Matching Fully Differential NNLO Calculations and Parton Showers, JHEP 06 (2014) 089 [arXiv: 1311.0286] [INSPIRE].

[69] S. Alioli, C.W. Bauer, C. Berggren, F.J. Tackmann and J.R. Walsh, Drell-Yan production at NNLL' + NNLO matched to parton showers, Phys. Rev. D 92 (2015) 094020 [arXiv: 1508.01475] [INSPIRE].

[70] S. Alioli, C.W. Bauer, S. Guns and F.J. Tackmann, Underlying event sensitive observables in Drell-Yan production using GENEVA, arXiv:1605.07192 [INSPIRE]. 
[71] S. Höche, Y. Li and S. Prestel, Drell-Yan lepton pair production at NNLO QCD with parton showers, Phys. Rev. D 91 (2015) 074015 [arXiv:1405.3607] [InSPIRE].

[72] S. Höche, Y. Li and S. Prestel, Higgs-boson production through gluon fusion at NNLO QCD with parton showers, Phys. Rev. D 90 (2014) 054011 [arXiv: 1407.3773] [inSPIRE].

[73] K. Hamilton, P. Nason and G. Zanderighi, MINLO: Multi-Scale Improved NLO, JHEP 10 (2012) 155 [arXiv: 1206.3572] [INSPIRE].

[74] J. Alwall et al., MadGraph/MadEvent v4: The New Web Generation, JHEP 09 (2007) 028 [arXiv: 0706.2334] [INSPIRE].

[75] J.M. Campbell, R.K. Ellis, R. Frederix, P. Nason, C. Oleari and C. Williams, NLO Higgs Boson Production Plus One and Two Jets Using the POWHEG BOX, MadGraph4 and MCFM, JHEP 07 (2012) 092 [arXiv:1202.5475] [INSPIRE].

[76] G. Cullen et al., GoSAm-2.0: a tool for automated one-loop calculations within the Standard Model and beyond, Eur. Phys. J. C 74 (2014) 3001 [arXiv:1404.7096] [InSPIRE].

[77] T. Melia, K. Melnikov, R. Rontsch and G. Zanderighi, Next-to-leading order QCD predictions for $W^{+} W^{+} j$ j production at the LHC, JHEP 12 (2010) 053 [arXiv: 1007.5313] [INSPIRE].

[78] NNPDF collaboration, R.D. Ball et al., Parton distributions for the LHC Run II, JHEP 04 (2015) 040 [arXiv:1410.8849] [INSPIRE].

[79] T. Sjöstrand, S. Mrenna and P.Z. Skands, PYTHIA 6.4 Physics and Manual, JHEP 05 (2006) 026 [hep-ph/0603175] [INSPIRE].

[80] T. Sjöstrand, S. Mrenna and P.Z. Skands, A Brief Introduction to PYTHIA 8.1, Comput. Phys. Commun. 178 (2008) 852 [arXiv:0710.3820] [INSPIRE].

[81] T. Sjöstrand et al., An Introduction to PYTHIA 8.2, Comput. Phys. Commun. 191 (2015) 159 [arXiv: 1410.3012] [INSPIRE].

[82] G. Luisoni, P. Nason, C. Oleari and F. Tramontano, $H W^{ \pm} / H Z+0$ and 1 jet at $N L O$ with the POWHEG BOX interfaced to GoSam and their merging within MiNLO, JHEP 10 (2013) 083 [arXiv: 1306.2542] [INSPIRE].

[83] M. Cacciari, G.P. Salam and G. Soyez, The Anti- $k_{\mathrm{T}}$ jet clustering algorithm, JHEP 04 (2008) 063 [arXiv: 0802.1189] [inSPIRE].

[84] M. Cacciari, G.P. Salam and G. Soyez, FastJet User Manual, Eur. Phys. J. C 72 (2012) 1896 [arXiv: 1111.6097] [inSPIRE]. 